Jan Lang*, Mathematical Institute, Academy of Sciences of the Czech Republic, Žitná 25, 11567 Prague 1, Czech Republic. e-mail:

lang@math.cas.cz

Aleš Nekvinda Thákurova 7, 16629 Praha 6, Czech Republic. e-mail:

nekvinda@fsv.cvut.cz

Jiří Rákosník ${ }^{\dagger}$, Mathematical Institute, Academy of Sciences of the Czech Republic, Žitná 25, 11567 Prague 1, Czech Republic. e-mail:

rakosnik@math.cas.cz

\title{
CONTINUOUS NORMS AND ABSOLUTELY CONTINUOUS NORMS IN BANACH FUNCTION SPACES ARE NOT THE SAME
}

\begin{abstract}
It is known that the concepts of continuous norm and of absolutely continuous norm do not coincide. There exists a space in which all functions possess continuous norm but not all functions possess absolutely continuous norm. In this paper we construct an extremal example of a Banach function space in which all functions have continuous norm but only the zero function has absolutely continuous norm.
\end{abstract}

\section{Introduction}

The concept of absolutely continuous norm plays a very important role in characterization of classes of reflexive Banach function spaces and of separable Banach function spaces. Let us recall two assertions presented by C. Bennett and R. Sharpley in [1]. The first one [1, Corollary 4.4] shows that a Banach function space $X$ is reflexive if and only if both $X$ and its associate space $X^{\prime}$

\footnotetext{
Key Words: Banach function space, absolutely continuous norm, continuous norm

Mathematical Reviews subject classification: 46E30

Received by the editors April 4, 2000

*The first author was supported by foundation "Nadání Josefa, Marie a Zdenky Hlávkových", Grants No. 201/96/0431 No. 201/98/PO17 of the Grant Agency of the Czech Republic.

${ }^{\dagger}$ The other authors were supported by Grant No. 201/97/0744 of the Grant Agency of the Czech Republic.
} 
have absolutely continuous norms (see also [7] and [8]). In the second one [1, Corollary 5.6] it is proved that a Banach function space $(X, \mu)$ is separable if and only if $X$ has absolutely continuous norm and $\mu$ is a separable measure. The concept of continuous norm was introduced by Q. Lai and L. Pick in [5]. They proved that the Hardy operator $T f(x)=\int_{0}^{x} f(t) d t$ is a compact mapping from a Banach function space $(X, v)$ into $L_{\infty}$ if and only if the function $1 / v$ has continuous norm in the associate space $\left(X^{\prime}, v\right)$. This result raised the question, if in every Banach function space $X$, the set of all functions with absolutely continuous norm is different from the set of all functions with continuous norm. In [6] two Banach function spaces are constructed which demonstrate that the concepts of continuous norm and absolutely continuous norm are not identical. The first space contains a function with continuous but not absolutely continuous norm and another function with non-continuous norm. In the other space, every function has continuous norm and there is a function with non-absolutely continuous norm.

D. E. Edmunds, J. Lang and A. Nekvinda [2] investigated the notions of continuous norm and absolutely continuous norm in the scale of spaces $L^{p(x)}$ of functions integrable with variable power $p(x)$. These spaces possess many non-standard properties (see [4], [3]). Nevertheless, the set of functions with continuous norm and the set of functions with absolutely continuous norm in these spaces coincide (see [2]).

In the present paper an extreme example of Banach function space is found in the sense that every function has continuous norm and only the zero function has absolutely continuous norm. The paper is organized in the following way. Section 2 has a preparatory character and brings basic notations, definitions and auxiliary assertions. In Section 3 we construct the Banach function space and in Section 4 we prove that it has the desired properties.

\section{Preliminaries}

Let $\Omega$ be a non-empty open subset of $\mathbb{R}$ and let $\mathcal{M}(\Omega)$ be the set of all real measurable functions defined on $\Omega$. Denote by $|E|$ the Lebesgue measure of any measurable subset $E$ of $\Omega$ and by $\chi_{E}$ the characteristic function of $E$. The unit function will be denoted $1_{\Omega}$; i.e., $1_{\Omega}(x)=1$ for all $x \in \Omega$.

Definition 2.1. A normed linear space $(X,\|\cdot\|)$ is called a Banach function space (abbreviated BFS) if the following conditions are satisfied:

(2.1) the norm $\|f\|$ is defined for all $f \in \mathcal{M}(\Omega)$, and $f \in X$ if and only if $\|f\|<\infty$;

$\|f\|=\||f|\|$ for every $f \in \mathcal{M}(\Omega)$ 
(2.3) if $0 \leq f_{n} \nearrow f$ a.e. in $\Omega$, then $\left\|f_{n}\right\| \nearrow\|f\|$;

(2.4) if $E \subset \Omega,|E|<\infty$, then $\chi_{E} \in X$;

(2.5) for every set $E \subset \Omega$ with $|E|<\infty$, there exists a positive constant $C_{E}$ such that $\int_{E}|f(x)| d x \leq C_{E}\|f\|$.

Recall that the Fatou property (2.3) immediately yields

(2.6) if $0 \leq f \leq g$, then $\|f\| \leq\|g\|$.

The above definition was introduced in [1]. In [8] another definition of Banach function spaces is given which-requiring properties (2.1), (2.2), (2.6) and the completeness of the space - specifies a wider class of spaces. The space we construct in this paper is a Banach function space in the sense of both definitions.

Definition 2.2. Let $X=(X(\Omega),\|\cdot\|)$ be a Banach function space and let $f \in X$ be an arbitrary function. We say that the function $f$ has absolutely continuous norm in $X$ if for any sequence of open sets $G_{n}$ with $G_{n} \supset G_{n+1}$ for $n=1,2, \ldots$ and $\bigcap_{n=1}^{\infty} G_{n}=\emptyset$, the norms $\left\|f \chi_{G_{n}}\right\|$ tend to zero as $n \rightarrow \infty$. The set of all functions with absolutely continuous norms is denoted by $X_{a}$.

We say that $f$ has continuous norm in $X$ if $\lim _{r \rightarrow 0+}\left\|f \chi_{(x-r, x+r) \cap \Omega}\right\|=0$ for every $x \in \bar{\Omega}$ and $\lim _{r \rightarrow \infty}\left\|f \chi_{\Omega \backslash(-r, r)}\right\|=0$. The set of all functions with continuous norm is denoted by $X_{c}$.

Recall that $\|\cdot\| \|: \mathcal{M}(\Omega) \rightarrow[-\infty, \infty]$ is a seminorm on $\mathcal{M}(\Omega)$ if every $f, g \in$ $\mathcal{M}(\Omega)$ and $a \in \mathbb{R}$ satisfy $\|f f|\|\geq 0,|\|a f|\|=|a||\|f\||\|,\|f+g\|| \leq|\|f|\|+\| g|\|$.

We will be particularly concerned with seminorms which satisfy conditions

$$
\||| f||=|||f||| \text { for all } f \in \mathcal{M}(\Omega)]
$$

(2.8) if $f_{n}, f \in \mathcal{M}(\Omega)$ and $0 \leq f_{n} \nearrow f$ a.e. in $\Omega$, then $\left\|f_{n}\right\|\|\nearrow\| f \|$.

Evidently, (2.8) implies

(2.9) if $0 \leq f \leq g$, then $\|f\| \leq\|\| g \|$.

We will need the following three assertions. The easy proofs are omitted.

Proposition 2.3. Let $\Omega_{i}, i \in I$, be a system of non-empty open subsets of $\Omega$ such that $\Omega=\bigcup_{i \in I} \Omega_{i} \cup M$, where $|M|=0$. Let $\|\cdot\|_{i} \|_{i}$ be a system of seminorms on $\Omega_{i}$ satisfying conditions (2.7) and (2.8). Define the seminorm $\|\mid\| \cdot \|$ by $\||f|\|=\sup _{i \in I} \mid\left\|f_{i}\right\|_{i}$, where $f_{i}$ denotes the restriction of $f$ on $\Omega_{i}$. Then || . || satisfies (2.7) and (2.8). 
Proposition 2.4. Let $\Omega_{n}$ be a sequence of non-empty open subsets of $\Omega$ and $\Omega=\bigcup_{n=1}^{\infty} \Omega_{n} \cup M$, where $|M|=0$. Let $\mid\|\cdot\|_{n}$ be a system of seminorms satisfying (2.7) and (2.8). Define the seminorm $\|f\|\left\|=\sum_{n=1}^{\infty}\right\|\left\|f_{n}\right\|_{n}$ for $f_{n}$ being the restriction of $f$ on $\Omega_{n}$. Then || $\mid$. || satisfies (2.7) and (2.8).

Proposition 2.5. Let $\||\|\mid\|$ be a seminorm satisfying (2.7) and (2.8) and let $\left\|1_{\Omega} \mid\right\|<\infty$. Define the norm $\|\cdot\|$ by $\|f\|=\left|\left\|f\left|\|+\int_{\Omega}\right| f \mid\right.\right.$. Then $\| \cdot \|$ is a norm in a $B F S$.

For the sake of simplicity, in the rest of the paper we assume $\Omega=(0,1)$.

\section{The Construction}

Our considerations are based on principles of construction of the Cantor set. Let the symbol $\mathcal{K}$ denote the set of all finite sequences of the numbers 0 and 1 including the empty sequence $\emptyset$. The elements of $\mathcal{K}$ will be called multiindices. The length $\ell(\alpha)$ of a multiindex $\alpha \in \mathcal{K}, \alpha=\left(a_{1}, a_{2}, \ldots, a_{n}\right)$, is the number of all members of the sequence $\alpha$; i.e., $\ell(\alpha)=n$. We define a partial ordering $\preceq$ on $\mathcal{K}$ saying that $\alpha \preceq \beta$ for $\alpha=\left(a_{1}, a_{2}, \ldots, a_{k}\right), \beta=\left(b_{1}, b_{2}, \ldots, b_{n}\right)$ if $k \leq n$ and $a_{i}=b_{i}$ for $i=1,2, \ldots, k$. We shall write $\alpha \npreceq \beta$ if $\alpha \preceq \beta$ does not hold, and $\alpha \nsim \beta$ if $\alpha \npreceq \beta$ and $\beta \npreceq \alpha$. Note, that $\alpha=\beta$ if $\alpha \preceq \beta$ and $\beta \preceq \alpha$, and that the relations $\nsim, \neq$ are different.

Recall the construction of the Cantor set $\mathfrak{C}$. Define the intervals $I_{\alpha}, \alpha \in \mathcal{K}$, 
by

$$
\begin{gathered}
I_{00}=\left(\frac{1}{3^{3}}, \frac{2}{3^{3}}\right) \\
I_{0}=\left(\frac{1}{3^{2}}, \frac{2}{3^{2}}\right) \\
I_{01}=\left(\frac{7}{3^{3}}, \frac{8}{3^{3}}\right) \\
I_{\emptyset}=\left(\frac{1}{3}, \frac{2}{3}\right) \quad I_{10}=\left(\frac{19}{3^{3}}, \frac{20}{3^{3}}\right) \\
I_{1}=\left(\frac{7}{3^{2}}, \frac{8}{3^{2}}\right) \\
I_{11}=\left(\frac{25}{3^{3}}, \frac{26}{3^{3}}\right)
\end{gathered}
$$

Note that $\left|I_{\alpha}\right|=3^{-\ell(\alpha)-1}$. The Cantor set is given by $\mathfrak{C}=[0,1] \backslash \bigcup_{\alpha \in \mathcal{K}} I_{\alpha}$. If $A, B \subset \mathbb{R}$ we say that $A$ is to the left (right) of $B$ if $\sup A<\inf B(\inf A>$ $\sup B)$, respectively. Let $\alpha, \beta \in \mathcal{K}$ and let $I_{\alpha}, I_{\beta}$ be the corresponding intervals from the above construction. We say that $\alpha$ is to the left (right) of $\beta$ if $I_{\alpha}$ is to the left (right) of $I_{\beta}$, respectively. If $\alpha$ is to the left of $\gamma$ and $\gamma$ is to the left of $\beta$, we say that $\gamma$ is between $\alpha$ and $\beta$. We denote by $\mathcal{F}$ the system of all bounded measurable sets $M \subset \mathbb{R}$ such that $|M \cap(x-t, x+t)|>0$ for every $x \in M$ and $t>0,|M|=3^{-n}$ for certain positive integer $n$, and, moreover, neither $\inf M$ nor $\sup M$ belong to $M$. Let $\mathcal{P}$ be the family of all mappings $P: \mathcal{K} \rightarrow \mathcal{F}$ such that $\bigcup_{\alpha \in \mathcal{K}} P(\alpha)$ is bounded,

$$
|P(\alpha)|=\frac{1}{3^{\ell(\alpha)+1}}
$$

and

if $\alpha$ is to the left of $\beta$, then $P(\alpha)$ is to the left of $P(\beta)$.

By $\mathcal{E}$ we denote the class of all measurable sets $E \subset \mathbb{R}$ such that $E=$ $\bigcup_{\alpha \in \mathcal{K}} P(\alpha)$ for some $P \in \mathcal{P}$. It is easy to see that for every $E \in \mathcal{E}$ the corresponding mapping $P \in \mathcal{P}$ is unique and $|E|=1$. We set $I_{\alpha}^{E}=P(\alpha)$, $\widetilde{I}_{\alpha}^{E}=\left(\inf I_{\alpha}^{E}, \sup I_{\alpha}^{E}\right)$. 
Note that the set $[0,1] \backslash \mathfrak{C}$ belongs to $\mathcal{E}$.

Let $E \in \mathcal{E}$. Let $P \in \mathcal{P}$ be the corresponding mapping and $I_{\alpha}^{E}=P(\alpha)$, $\alpha \in \mathcal{K}$. For $\alpha \in \mathcal{K}$ we define the closed intervals $J_{\alpha}^{E}$ by

$$
\begin{array}{ll} 
& J_{00}^{E}=\left[\inf E, \inf I_{0}^{E}\right] \\
J_{0}^{E}=\left[\inf E, \inf I_{\emptyset}^{E}\right] & \\
J_{\emptyset}^{E}=[\inf E, \sup E] & J_{01}^{E}=\left[\sup I_{0}^{E}, \inf I_{\emptyset}^{E}\right] \\
& J_{10}^{E}=\left[\sup I_{\emptyset}^{E}, \inf I_{1}^{E}\right] \\
J_{1}^{E}=\left[\sup I_{\emptyset}^{E}, \sup E\right] & \\
& J_{11}^{E}=\left[\sup I_{1}^{E}, \sup E\right]
\end{array}
$$

The generalized Cantor set $\mathfrak{C}^{E}$ corresponding to the set $E$ is defined by

$$
\mathfrak{C}^{E}=\bigcap_{n=1}^{\infty} \bigcup_{\ell(\alpha)=n} J_{\alpha}^{E}
$$

It is not difficult to show the following properties:

$$
\left|J_{\alpha}^{E}\right| \geq\left|J_{\alpha}^{E} \cap E\right|=\sum_{\alpha \preceq \beta}\left|I_{\beta}^{E}\right|=3^{-\ell(\alpha)}
$$

(3.4) the families $\left\{I_{\alpha}^{E}\right\}_{\alpha}$ and $\left\{\widetilde{I}_{\alpha}^{E}\right\}_{\alpha}$ are pairwise disjoint;

(3.5) $\alpha \preceq \beta$ if and only if $J_{\beta}^{E} \subset J_{\alpha}^{E}$ and $\alpha \nsim \beta$ if and only if $J_{\alpha}^{E} \cap J_{\beta}^{E}=\emptyset$;

(3.6) $\alpha \preceq \beta$ if and only if $\widetilde{I}_{\beta}^{E} \subset J_{\alpha}^{E}$ and $\alpha \npreceq \beta$ if and only if $\widetilde{I}_{\beta}^{E} \cap J_{\alpha}^{E}=\emptyset$;

(3.7) if $\alpha \in \mathcal{K}$ and if $k$ is an integer, $k \geq \ell(\alpha)$, then the number of all multiindices $\beta$ such that $\alpha \preceq \beta$ and $\ell(\beta)=k$, is equal to $2^{k-\ell(\alpha)}$; 
(3.8) for every $x \in \mathfrak{C}^{E}$ there exists a unique sequence $\left\{\alpha_{n}\right\}_{n=1}^{\infty}$ such that $\alpha_{1} \preceq \alpha_{2} \preceq \ldots, \ell\left(\alpha_{n}\right)=n$ and $x \in \bigcap_{n=1}^{\infty} J_{\alpha_{n}}^{E}$.

Moreover, if $\alpha$ is to the left of $\beta$ and $\alpha \nsim \beta$, then there exists $\gamma$ between $\alpha$ and $\beta$ such that $\gamma \preceq \alpha$ and $\gamma \preceq \beta$.

Now, we are ready to construct the desired norm in a BFS. Consider the class $\mathcal{M}=\mathcal{M}(0,1)$. We will identify the functions $f \in \mathcal{M}$ with their extensions by zero outside $(0,1)$. For $E \in \mathcal{E}$ we define the seminorm $\|\mid\|\|\|_{E}$ by

$$
\begin{aligned}
\|f\|_{E} & =\sum_{k=0}^{\infty} 2^{-k} \max _{\ell(\alpha)=k} \sup _{n \geq k}\left|\bigcup I_{\beta}^{E}\right|^{-1} \int_{\bigcup I_{\beta}^{E}}|f(x)| d x \\
& =\sum_{k=0}^{\infty} \max _{\ell(\alpha)=k} \sup _{n \geq k} \frac{3^{n+1}}{2^{n}} \sum_{\substack{\ell(\beta)=n \\
\alpha \preceq \beta}} \int_{I_{\beta}^{E}}|f(x)| d x, \quad f \in \mathcal{M},
\end{aligned}
$$

where the union is taken over all $\beta$ such that $\ell(\beta)=n$ and $\alpha \preceq \beta$; the second equality follows from (3.3).

Definition 3.1. The space $X$ is the set of all functions $f \in \mathcal{M}$ with $\|f\|<\infty$ where $\|f\|=\sup _{E \in \mathcal{E}}\|f\|_{E}$.

Theorem 3.2. $(X,\|\cdot\|)$ is a BFS.

Proof. Set $\|f\|_{0}=\|f\|+\int_{0}^{1}|f|$. Considering the only term with $k=n=0$ on the right hand side of (3.9) we obtain $\|f\|_{E} \geq 3 \int_{I_{\emptyset}^{E}}|f(x)| d x$ for every $E \in \mathcal{E}$. If $E_{1}, E_{2}, E_{3} \in \mathcal{E}$ are such that $I_{\emptyset}^{E_{1}}=(0,1 / 3), I_{\emptyset}^{E_{2}}=(1 / 3,2 / 3)$, $I_{\emptyset}^{E_{3}}=(2 / 3,1)$, then there exists $i \in\{1,2,3\}$ such that $3 \int_{I_{\emptyset}^{E_{i}}}|f| \geq \int_{0}^{1}|f|$. Hence

$$
\|f\| \geq\left|\left\|f\left|\|_{E_{i}} \geq 3 \int_{I_{\emptyset}^{E_{i}}}\right| f\left|\geq \int_{0}^{1}\right| f \mid,\right.\right.
$$

and so, $2\|f\| \geq\|f\|_{0} \geq\|f\|$. It suffices to show that $\|\cdot\|_{0}$ is a norm in a BFS.

According to Propositions 2.3 and 2.4 every seminorm $\|\cdot\| \|_{E}, E \in \mathcal{E}$, satisfies conditions $(2.7)$ and $(2.8)$ on the interval $(0,1)$. Repeated application of Proposition 2.3 yields that the seminorm $\|f\|=\sup _{E \in \mathcal{E}}\|f\|_{E}$ satisfies (2.7) and (2.8). By Proposition 2.5, it remains to verify that the unit function $1_{\Omega}$ satisfies $\left\|1_{\Omega}\right\|<\infty$ which is seen from (3.9). $\left\|1_{\Omega}\right\|_{E} \leq \sum_{k=0}^{\infty} 2^{-k}=2$ for every $E \in \mathcal{E}$; i.e., $\left\|1_{\Omega}\right\| \leq 2<\infty$. 


\section{Properties of the Space $X$}

In this section we prove two theorems. The first one claims that only the zero function in $X$ has absolutely continuous norm; i.e., $X_{a}=\{0\}$, while the other claims that every function from $X$ has continuous norm; i.e., $X_{c}=X$.

Theorem 4.1. $X_{a}=\{0\}$.

Proof. Let $f \in X, f \neq 0$. Then there exist a measurable set $M,|M|>0$, and a real number $\eta>0$ such that $|f| \geq \eta>0$ on $M$. Set

$$
M^{\prime}=\{x \in M:|(x-t, x+t) \cap M|>0 \text { for every } t>0\} .
$$

Clearly, $\left|M^{\prime}\right|=|M|$. Take the integer $n_{0}$ such that $3^{-n_{0}-1} \leq\left|M^{\prime}\right|<3^{-n_{0}}$ and the real number $b<1$ such that $\left|\left[\inf M^{\prime}, b\right] \cap M^{\prime}\right|=3^{-n_{0}-1}$. Set

$$
F_{1}=\left[\inf M^{\prime}, b\right] \cap M^{\prime}, F_{2}=\left[1,2-3^{-n_{0}-1}\right] \text {, and } F=F_{1} \cup F_{2} .
$$

For every $\alpha \in \mathcal{K}$ we denote by $p(\alpha), q(\alpha)$ the least real numbers such that $|(\inf F, p(\alpha)) \cap F|=\inf I_{\alpha},|(p(\alpha), q(\alpha)) \cap F|=\left|I_{\alpha}\right|$, and we set $P(\alpha)=$ $(p(\alpha), q(\alpha)) \cap F$. It is easy to see that $P \in \mathcal{P}$ and so the set $E=\bigcup_{\alpha \in \mathcal{K}} P(\alpha)$ belongs to $\mathcal{E}$. Set $E_{1}=E \cap F_{1}, E_{2}=E \cap F_{2}$ and $\alpha_{0}=(0,0, \ldots, 0), \ell\left(\alpha_{0}\right)=n_{0}$. Since $E_{1}$ is to the left of $E_{2}$ and $\left|E_{1}\right|=3^{-n_{0}-1}$, we have $I_{\beta}^{E} \cap F_{1}=I_{\beta}^{E}$ if $\beta$ is to the left of $\alpha_{0}$ and $I_{\beta}^{E} \cap F_{1}=\emptyset$ in other cases. For every non-negative integer $N$ we set $G_{N}=\bigcup_{\ell(\alpha) \geq N} \widetilde{I}_{\alpha}^{E}$. Obviously, $G_{N}$ form a decreasing sequence of open sets with empty intersection. Let us calculate $\left\|f \chi_{G_{N}}\right\|_{E}$ for a fixed $N$. Taking only the first summand in (3.9), using (3.4) and the fact that $\widetilde{I}_{\alpha}^{E} \cap E=I_{\alpha}^{E}$, 
we obtain

$$
\begin{aligned}
\left\|f \chi_{G_{N}}\right\|_{E} & \geq \sup _{n \geq 0} \frac{3^{n+1}}{2^{n}} \sum_{\ell(\beta)=n} \int_{I_{\beta}^{E}}\left|f \chi_{G_{N}}\right| \\
& =\sup _{n \geq 0} \frac{3^{n+1}}{2^{n}} \sum_{\ell(\beta)=n} \int_{I_{\beta}^{E}}|f| \sum_{\ell(\alpha) \geq N} \chi_{\widetilde{I}_{\alpha}^{E}} \\
& \geq \sup _{n \geq 0} \frac{3^{n+1}}{2^{n}} \sum_{\ell(\beta)=n} \int_{I_{\beta}^{E}}|f| \sum_{\ell(\alpha) \geq N} \chi_{\widetilde{I}_{\alpha}^{E} \cap E} \\
& =\sup _{n \geq 0} \frac{3^{n+1}}{2^{n}} \sum_{\ell(\beta)=n} \sum_{(\alpha) \geq N} \int_{I_{\beta}^{E}}\left|f \chi_{I_{\alpha}^{E}}\right| \\
& \geq \sup _{n \geq N} \frac{3^{n+1}}{2^{n}} \sum_{\ell(\beta)=n} \sum_{\ell(\alpha)=n} \int_{I_{\beta}^{E}}\left|f \chi_{I_{\alpha}^{E}}\right| \\
& \geq \sup _{n \geq N} \frac{3^{n+1}}{2^{n}} \sum_{\ell(\beta)=n} \int_{I_{\beta}^{E} \cap E_{1}}|f| .
\end{aligned}
$$

By (3.7) and the inclusion $E_{1} \subset M$ we arrive at

$$
\left\|f \chi_{G_{N}}\right\| \|_{E} \geq \sup _{n \geq N} \frac{3^{n+1}}{2^{n}} \int_{\cup I_{\beta}^{E}}|f| \geq \sup _{n \geq N} \frac{3^{n+1}}{2^{n}} \frac{2^{n-\ell\left(\alpha_{0}\right)-1}}{3^{n+1}} \eta=\frac{\eta}{2^{n_{0}+1}}
$$

where the union is taken over all $\beta$ left of $\alpha_{0}$ such that $\ell(\beta)=n$. Thus,

$$
\left\|f \chi_{G_{N}}\right\|=\sup _{E \in \mathcal{E}}\left\|f \chi_{G_{N}}\right\|_{E} \geq \frac{\eta}{2^{n_{0}+1}}
$$

and therefore $f$ does not have absolutely continuous norm.

The proof of $X_{c}=X$ is rather technical. The core is contained in the following five lemmas.

Lemma 4.2. Let $E \in \mathcal{E}$, let $\alpha_{1} \preceq \alpha_{2} \preceq \ldots \preceq \alpha_{j} \preceq \ldots$ be a sequence of multiindices with $\ell\left(\alpha_{j}\right)=j$ and let $\|f \mid\|_{E}<\infty$. Then

$$
\lim _{j \rightarrow \infty}\left\|f \chi_{J_{\alpha_{j}}^{E}}\right\| \|_{E}=0 .
$$

Proof. It follows from (3.5) and (2.9) that $\left\|f \chi_{J_{\alpha_{j}}^{E}}\right\| \|_{E}$ is non-increasing. Assume that (4.1) does not hold. Then there exists $\eta>0$ such that for every $j$ the inequality

$$
\left\|f \chi_{J_{\alpha_{j}}^{E}} \mid\right\|_{E} \geq \eta
$$


holds. Let us fix $j$ and calculate $\left\|f \chi_{J_{\alpha_{j}}^{E}}\right\| \|_{E}$. According to (3.9) we have

$$
\begin{aligned}
\left\|f \chi_{J_{\alpha_{j}}^{E}} \mid\right\|_{E}= & \sum_{k=0}^{j-1} \max _{\ell(\alpha)=k} \sup _{n \geq k} \frac{3^{n+1}}{2^{n}} \sum_{\substack{\ell(\beta)=n \\
\alpha \preceq \beta}} \int_{I_{\beta}^{E} \cap J_{\alpha_{j}}^{E}}|f| \\
& +\sum_{k=j}^{\infty} \max _{\ell(\alpha)=k} \sup _{n \geq k} \frac{3^{n+1}}{2^{n}} \sum_{\substack{\ell(\beta)=n \\
\alpha \preceq \beta}} \int_{I_{\beta}^{E} \cap J_{\alpha_{j}}^{E}}|f| \\
& =A_{j}+B_{j} .
\end{aligned}
$$

Let $0 \leq k \leq j-1$. Then $\alpha_{k} \preceq \alpha_{j}$. If $\alpha \preceq \beta, \ell(\alpha)=k$ and $\alpha \nsim \alpha_{k}$, then $\beta \nsim \alpha_{j}$ and, according to (3.6), $I_{\beta}^{E} \cap J_{\alpha_{j}}^{E}=\emptyset$. Therefore,

$$
\max _{\substack{\ell(\alpha)=k \\ \alpha \nsim \alpha_{k}}} \sup _{n \geq k} \frac{3^{n+1}}{2^{n}} \sum_{\substack{\ell(\beta)=n \\ \alpha \preceq \beta}} \int_{I_{\beta}^{E} \cap J_{\alpha_{j}}^{E}}|f|=0
$$

and we can rewrite $A_{j}$ in the following form.

$$
A_{j}=\sum_{k=0}^{j-1} \sup _{n \geq k} \frac{3^{n+1}}{2^{n}} \sum_{\substack{\ell(\beta)=n \\ \alpha_{k} \preceq \beta}} \int_{I_{\beta}^{E} \cap J_{\alpha_{j}}^{E}}|f| .
$$

If $k \leq n \leq j-1$, then the inequalities $\ell(\beta) \leq j-1<j=\ell\left(\alpha_{j}\right)$ imply $\alpha_{j} \npreceq \beta$ and (3.6) yields $I_{\beta}^{E} \cap J_{\alpha_{j}}^{E}=\emptyset$. Thus, we can write

$$
A_{j}=\sum_{k=0}^{j-1} \sup _{n \geq j} \frac{3^{n+1}}{2^{n}} \sum_{\substack{\ell(\beta)=n \\ \alpha_{k} \preceq \beta}} \int_{\substack{I_{\beta}^{E} \cap J_{\alpha_{j}}^{E} \\ \mid}}|f| .
$$

If $n \geq j$, then again (3.6) implies that $I_{\beta}^{E} \cap J_{\alpha_{j}}^{E}=\emptyset$ if $\alpha_{j} \npreceq \beta$ and $I_{\beta}^{E} \cap J_{\alpha_{j}}^{E}=I_{\beta}^{E}$ if $\alpha_{j} \preceq \beta$. Hence, we can further reduce the expression of $A_{j}$.

$$
A_{j}=\sum_{k=0}^{j-1} \sup _{n \geq j} \frac{3^{n+1}}{2^{n}} \sum_{\substack{\ell(\beta)=n \\ \alpha_{j} \preceq \beta}} \int_{I_{\beta}^{E}}|f|=j \sup _{n \geq j} \frac{3^{n+1}}{2^{n}} \sum_{\substack{\ell(\beta)=n \\ \alpha_{j} \preceq \beta}} \int_{I_{\beta}^{E}}|f| .
$$

Since

$$
B_{j} \leq \sum_{k=j}^{\infty} \max _{\ell(\alpha)=k} \sup _{n \geq k} \frac{3^{n+1}}{2^{n}} \sum_{\substack{\ell(\beta)=n \\ \alpha \preceq \beta}} \int_{I_{\beta}^{E}}|f| \leq\|f \mid\|_{E}<\infty
$$


we have $\lim _{j \rightarrow \infty} B_{j}=0$, and so, according to (4.2), (4.3), there exists $j_{0}$ such that $A_{j} \geq \eta / 2$ for $j \geq j_{0}$ and, by (4.4),

$$
\sup _{n \geq j} \frac{3^{n+1}}{2^{n}} \sum_{\substack{\ell(\beta)=n \\ \alpha_{j} \preceq \beta}} \int_{I_{\beta}^{E}}|f| \geq \frac{\eta}{2 j}, \quad j \geq j_{0} .
$$

Thus, using the assumption $\ell\left(\alpha_{k}\right)=k$, we obtain

$$
\left\|f\left|\|_{E} \geq \sum_{k=j_{0}}^{\infty} \sup _{n \geq k} \frac{3^{n+1}}{2^{n}} \sum_{\substack{\ell(\beta)=n \\ \alpha_{k} \preceq \beta}} \int_{I_{\beta}^{E}}\right| f \mid \geq \frac{\eta}{2} \sum_{k=j_{0}}^{\infty} \frac{1}{k}=\infty,\right.
$$

which contradicts the assumption.

Lemma 4.3. Let $E \in \mathcal{E}$ and $\|f\|_{E}<\infty$. Then $\lim _{t \rightarrow 0+}\left\|f \chi_{(x-t, x+t)}\right\| \|_{E}=0$ for all $x \in \mathbb{R}$.

Proof. We can assume that $x \in[\inf E, \sup E]$.

First of all, consider the case $x \notin \mathfrak{C}^{E}$. By (3.4), there exists a unique multiindex $\gamma \in \mathcal{K}$ such that $(x-t, x+t) \subset \widetilde{I}_{\gamma}^{E}$ for sufficiently small $t>0$, and so

$$
\begin{aligned}
\left\|f \chi_{(x-t, x+t)} \mid\right\|_{E} & =\left\|f \chi_{(x-t, x+t) \cap \widetilde{I}_{\gamma}^{E}}\right\| \|_{E} \\
& =\sum_{k=0}^{\infty} \max _{\ell(\alpha)=k} \sup _{n \geq k} \frac{3^{n+1}}{2^{n}} \sum_{\substack{\ell(\beta)=n \\
\alpha \preceq \beta}} \int_{I_{\beta}^{E} \cap \widetilde{I}_{\gamma}^{E}}\left|f \chi_{(x-t, x+t)}\right| .
\end{aligned}
$$

Property (3.4) yields

$$
\sup _{n \geq k} \frac{3^{n+1}}{2^{n}} \sum_{\substack{\ell(\beta)=n \\ \alpha \preceq \beta}} \int_{I_{\beta}^{E} \cap \widetilde{I}_{\gamma}^{E}}\left|f \chi_{(x-t, x+t)}\right|= \begin{cases}\frac{3^{\ell(\gamma)+1}}{2^{\ell(\gamma)}} \int_{I_{\gamma}^{E}}\left|f \chi_{(x-t, x+t)}\right| & \text { if } \alpha \preceq \gamma, \\ 0 & \text { if } \alpha \npreceq \gamma .\end{cases}
$$

Hence

$$
\left\|f f \chi_{(x-t, x+t)}\left|\|_{E}=\sum_{k=0}^{\ell(\gamma)} \frac{3^{\ell(\gamma)+1}}{2^{\ell(\gamma)}} \int_{I_{\gamma}^{E} \cap(x-t, x+t)}\right| f \mid \rightarrow 0 \text { as } t \rightarrow 0+,\right.
$$

since $\int_{I_{\gamma}^{E}}|f| \leq\|f \mid\|_{E}<\infty$. 
Now, assume that $x \in \mathfrak{C}^{E}$. Let $\left\{\alpha_{n}\right\}$ be the sequence of multiindices from (3.8). If $x$ is an inner point of all $J_{\alpha_{n}}^{E}$, then there is a sequence of positive real numbers $t_{n}$ such that $\left(x-t_{n}, x+t_{n}\right) \subset J_{\alpha_{n}}^{E}$ and for $0<t<t_{n}$ we have

$$
\left\|f \chi_{(x-t, x+t)}\right\|\left\|_{E} \leq\right\| f \chi_{J_{\alpha_{n}}^{E}}\|\|_{E} .
$$

By Lemma 4.2 the last term tends to 0 as $n \rightarrow \infty$. So $\lim _{t \rightarrow 0+}\left\|f \chi_{(x-t, x+t)} \mid\right\|_{E}=0$.

If $x=\inf E$ or $x=\sup E$, then $(x-t, x+t) \cap E$ is $(x, x+t) \cap E$ or $(x-t, x) \cap E$ and we arrive at (4.5) replacing $(x-t, x+t)$ with $(x, x+t)$ or $(x-t, x)$ respectively.

Finally, if there is $n$ such that $x$ is one of the end points of $J_{\alpha_{n}}^{E}$ and $x \neq \inf E, x \neq \sup E$, then there exists a unique interval $\widetilde{I}_{\alpha}^{E}$ such that either $x=\inf \widetilde{I}_{\alpha}^{E}$ or $x=\sup \widetilde{I}_{\alpha}^{E}$. We will investigate only the case $x=\inf \widetilde{I}_{\alpha}^{E}$. The other case is analogous. Then $x=\sup J_{\alpha_{j}}^{E}$ for all $\alpha_{j}$ with $\ell\left(\alpha_{j}\right) \geq \ell(\alpha)+1$, and we can write

$$
\begin{aligned}
\left\|f \chi_{(x-t, x+t)} \mid\right\|_{E} & \leq\left\|f \chi_{(x-t, x)}\right\|_{E}+\left\|f \chi_{(x, x+t)}\right\|_{E} \\
& =\left\|f \chi_{(x-t, x) \cap J_{\alpha_{j}}^{E}}\right\|_{E}+\left\|\chi_{(x, x+t) \cap I_{\alpha}^{E}}\right\|_{E}
\end{aligned}
$$

for such $t$ that $(x-t, x) \subset J_{\alpha_{j}}^{E}$ and $(x, x+t) \subset \widetilde{I}_{\alpha}^{E}$. The first term can be estimated in the same way as (4.5). The second term can be treated as the case $x \notin \mathfrak{C}^{E}$.

For $r \in \mathbb{R}$ and $M \subset \mathbb{R}$ we define the set $r+M$ by

$$
r+M=\{r+x: x \in M\} .
$$

Lemma 4.4. Let $0<t<1,0<\tau \leq \eta / 4$ and let $f \in X$ satisfy

$$
\sup _{E \in \mathcal{E}}\left\|f \chi_{(0, t)} \mid\right\|_{E} \geq \eta \text {. }
$$

Then there exists $E \in \mathcal{E}$ and $\gamma \in \mathcal{K}$ such that

$$
\begin{gathered}
0<s:=\inf I_{\gamma}^{E}<t, \\
|| f \chi_{(s, t)} \|_{E} \geq \frac{\eta}{2}-2 \tau, \\
\sup I_{\omega}^{E} \leq 4 t \quad \text { and } \quad\left|I_{\omega}^{E}\right| \leq 3 t \quad \text { whenever } I_{\omega}^{E} \cap(s, t) \neq \emptyset .
\end{gathered}
$$

Proof. Take $E_{0} \in \mathcal{E}$ such that

$$
\eta-\tau \leq\|\| f \chi_{(0, t)}\left|\left\|_{E_{0}} \leq \sup _{E \in \mathcal{E}}\right\| f \chi_{(0, t)}\right| \|_{E} .
$$


At first, we will find $E_{1} \in \mathcal{E}$ and $\gamma \in \mathcal{K}$ such that (4.7) and (4.8) hold with $E_{1}$ in place of $E$. According to Lemma 4.3, there is a number $s_{0}, 0<s_{0}<t$, such that $\left\|f \chi_{\left(0, s_{0}\right)} \mid\right\|_{E_{0}} \leq \tau$ and so, by (4.10),

$$
\left\|f \chi_{\left(s_{0}, t\right)} \mid\right\|_{E_{0}} \geq \eta-2 \tau \text {. }
$$

Set $r=\inf \bigcup I_{\beta}^{E_{0}}$ where the union is taken over all $\beta \in \mathcal{K}$ such that

$$
I_{\beta}^{E_{0}} \cap\left(s_{0}, t\right) \neq \emptyset .
$$

Note, that $r<t$. We will distinguish two cases: $s_{0} \leq r$ and $r<s_{0}$. Let $s_{0} \leq r$. Then $\left\|f \chi_{\left(s_{0}, t\right)} \mid\right\|_{E_{0}}=\left\|f \chi_{(r, t)}\right\|_{E_{0}}$. By Lemma 4.3, there exists a number $t_{1}$, $r<t_{1}<t$, such that $\left\|f \chi_{\left(r, t_{1}\right)}\right\|_{E_{0}}<\tau$. We take $\gamma$ such that $r \leq \inf I_{\gamma}^{E_{0}}<t_{1}$. Then $\gamma$ satisfies (4.11). We define the mapping $P: \mathcal{K} \rightarrow \mathcal{F}$ by

$$
P(\omega)= \begin{cases}I_{\omega}^{E_{0}} & \text { if } \omega=\gamma \text { or if } \omega \text { is to the right of } \gamma \\ -1+I_{\omega} & \text { if } \omega \text { is to the left of } \beta\end{cases}
$$

where $I_{\omega}$ are the intervals from the construction of the classical Cantor set in Section 3 and we set $E_{1}=\bigcup_{\omega \in \mathcal{K}} P(\omega)$. Clearly, $P \in \mathcal{P}$ and $E_{1} \in \mathcal{E}$. Then (4.7) is satisfied and the estimates

$$
\left.\left\|f \chi_{(s, t)}\right\|\right|_{E_{1}} \geq\left\|f \chi_{\left(t_{1}, t\right)}\left|\left\|_{E_{0}} \geq\right\| f \chi_{(r, t)}\right|\right\|_{E_{0}}-\||| f \chi_{\left(r, t_{1}\right)}||_{E_{0}} \geq \eta-3 \tau
$$

yield

$$
\left\|f \chi_{(s, t)}\right\|_{E_{1}}>\frac{\eta}{2}-2 \tau .
$$

Now, let $r<s_{0}$. There exists a unique multiindex $\beta$ satisfying (4.11) and $\inf I_{\beta}^{E_{0}}<s_{0}$. Then $\inf I_{\beta}^{E_{0}}=r$ and either

$$
r<s_{0}<t \leq q
$$

or

$$
r<s_{0}<q<t
$$

where $q=\sup I_{\beta}^{E_{0}}$.

Assume first that (4.13) holds. We change the set $E_{0}$ pasting the mass of $I_{\beta}^{E_{0}} \backslash\left(s_{0}, t\right)$ to the right of $t$. We define the mapping $P: \mathcal{K} \rightarrow \mathcal{F}$ by

$$
P(\omega)= \begin{cases}{\left[\left(s_{0}, t\right) \cap I_{\beta}^{E_{0}}\right] \cup\left(t, t+\left|I_{\beta}^{E_{0}} \backslash\left(s_{0}, t\right)\right|\right)} & \text { if } \omega=\beta, \\ -1+I_{\omega} & \text { if } \omega \text { is to the left of } \beta \\ t+\left|I_{\beta}^{E_{0}} \backslash\left(s_{0}, t\right)\right|+I_{\omega} & \text { if } \omega \text { is to the right of } \beta\end{cases}
$$


and set $E_{1}=\bigcup_{\omega \in \mathcal{K}} P(\omega)$. Clearly, $P \in \mathcal{P}$ and $E_{1} \in \mathcal{E}$. We set $\gamma=\beta$. Since $\left(s_{0}, t\right) \cap E_{1}=\left(s_{0}, t\right) \cap E_{0}=\left(s_{0}, t\right) \cap I_{\beta}^{E_{0}}=\left(s_{0}, t\right) \cap I_{\beta}^{E_{1}}=(s, t) \cap I_{\beta}^{E_{1}}$, we have

$$
\left\|f \chi_{(s, t)}\left|\left\|_{E_{1}}=\right\|\right| \mid f \chi_{\left(s_{0}, t\right)}\right\|_{E_{0}} \geq \eta-2 \tau>\frac{\eta}{2}-2 \tau .
$$

Now, let (4.14) hold. Then

$$
\eta-2 \tau \leq\left|\left\|f \chi _ { ( s _ { 0 } , t ) } \left|\left\|_{E_{0}} \leq\left.\left|\left\|f \chi_{\left(s_{0}, q\right)}\right\|_{E_{0}}+\|\| f \chi_{(q, t)}\right|\right|_{E_{0}}=A+B\right.\right.\right.\right.
$$

and so, either $A \geq \frac{\eta}{2}-\tau$ or $B \geq \frac{\eta}{2}-\tau$. If $A \geq \frac{\eta}{2}-\tau$ we define $P$ by

$$
P(\omega)= \begin{cases}-1+I_{\omega} & \text { if } \omega \text { is to the left of } \beta, \\ {\left[\left(s_{0}, q\right) \cap E_{0}\right] \cup\left(q, q+\left|\left(r, s_{0}\right) \cap E_{0}\right|\right)} & \text { if } \omega=\beta, \\ q+\left|\left(r, s_{0}\right) \cap E_{0}\right|+I_{\omega} & \text { if } \omega \text { is to the right of } \beta,\end{cases}
$$

and set $E_{1}=\bigcup_{\omega \in \mathcal{K}} P(\omega)$. As in the previous case, $P \in \mathcal{P}$, setting $\gamma=\beta$ we obtain (4.7), and

$$
\left\|f \chi_{(s, t)}\left|\left\|_{E_{1}} \geq\right\| f \chi_{(s, q)}\right|\right\|_{E_{1}}=\left.\left\|f \chi_{\left(s_{0}, q\right)}\right\|\right|_{E_{0}}=A \geq \frac{\eta}{2}-\tau
$$

implies (4.12).

If $B \geq \frac{\eta}{2}-\tau$, we set $t_{1}=\inf \bigcup I_{\omega}^{E_{0}}$ where the infimum is taken over all $\omega \in \mathcal{K}$, which are to the right of $\beta$. Then $q<t_{1}<t$. By Lemma 4.3, there is a number $s_{2}, t_{1}<s_{2}<t$, such that $\left\|f \chi_{\left(s_{2}, t\right)}\right\|_{E_{0}} \geq \frac{\eta}{2}-2 \tau$. According to the definition of $t_{1}$ there is a multiindex $\beta^{\prime}$ such that $t_{1} \leq \inf I_{\beta^{\prime}}^{E_{0}}<s_{2}$. We define

$$
P(\omega)= \begin{cases}-1+I_{\omega} & \text { if } \omega \text { is to the left of } \beta^{\prime}, \\ I_{\beta^{\prime}}^{E_{0}} & \text { if } \omega=\beta^{\prime}, \\ I_{\omega}^{E_{0}} & \text { if } \omega \text { is to the right of } \beta^{\prime} .\end{cases}
$$

Then $P \in \mathcal{P}$, and setting $E_{1}=\bigcup_{\omega \in \mathcal{K}} P(\omega)$ and $\gamma=\beta^{\prime}$ we obtain (4.7) and also (4.12) since

$$
\left\|f \chi_{(s, t)}||_{E_{1}} \geq\right\| f \chi_{\left(s_{2}, t\right)} \mid \|_{E_{0}} .
$$

Thus, we have $E_{1} \in \mathcal{E}$ and $\gamma \in \mathcal{K}$ satisfying (4.7) and (4.8). If $E_{1}$ satisfies (4.9), we set $E=E_{1}$. Assume that $E_{1}$ does not satisfy (4.9). We have to change it so that (4.7), (4.8) remain valid and, moreover, (4.9) holds.

If $\left|I_{\omega}^{E_{1}}\right| \leq 3 t$ for all $\omega$ such that $I_{\omega}^{E_{1}} \cap(s, t) \neq \emptyset$, then there exists $\alpha \in \mathcal{K}$ such that $\inf I_{\alpha}^{E_{1}}<t<\sup I_{\alpha}^{E_{1}}$. We define

$$
P(\omega)= \begin{cases}-1+I_{\omega} & \text { if } \omega \text { is to the left of } \alpha, \\ {\left[I_{\alpha}^{E_{1}} \cap(s, t)\right] \cup\left(t, t+\left|I_{\alpha}^{E_{1}} \backslash(s, t)\right|\right)} & \text { if } \omega=\alpha, \\ 5 t+I_{\omega} & \text { if } \omega \text { is to the right of } \alpha\end{cases}
$$


and set $E=\bigcup_{\omega \in \mathcal{K}} P(\omega)$. It is seen that (4.9) holds.

If $\left|I_{\alpha}^{E_{1}}\right|>3 t$ and $I_{\alpha}^{E_{1}} \cap(s, t) \neq \emptyset$ for some $\alpha \in \mathcal{K}$, then this $\alpha$ is determined in a unique way. We take the integer $j$ such that

$$
3^{j} t<\left|I_{\alpha}^{E_{1}}\right| \leq 3^{j+1} t
$$

and denote by $\mathcal{L}$ the set of all $\omega \in \mathcal{K}$ such that $I_{\omega}^{E_{1}} \cap(s, t) \neq \emptyset$. We denote $\alpha=\left(a_{1}, \ldots, a_{k}\right)$ and define the multiindex $\alpha^{\prime}=\left(a_{1}, \ldots, a_{k}, 0,1, \ldots, 1\right)$ with $\ell\left(\alpha^{\prime}\right)=k+j$. The family $\mathcal{L}$ has the properties

$$
\alpha^{\prime} \notin \mathcal{L}
$$

if $\omega$ is between $\omega_{1}, \omega_{2} \in \mathcal{L}$, then $\omega \in \mathcal{L}$,

if $\omega \in \mathcal{L} \backslash\{\alpha\}$, then $\alpha^{\prime} \preceq \omega, \ell(\omega)>\ell\left(\alpha^{\prime}\right)$ and $\omega$ is to the right of $\alpha^{\prime}$.

We set $z=3^{-k-j-1}-\left|I_{\alpha}^{E_{1}} \cap(s, t)\right|$. Note that (4.15) yields $z>0$. It follows from (4.16) that the $(k+j+1)$-th component of every $\omega \in \mathcal{L} \backslash\{\alpha\}$ is 1 . We consider the injection $\kappa: \mathcal{L} \backslash\{\alpha\} \rightarrow \mathcal{K}$ which changes the $(k+j+1)$-th component from 1 to 0 and keeps all other components unchanged. Obviously, $\kappa$ preserves the relation $\preceq$. We define the mapping $P: \mathcal{K} \rightarrow \mathcal{F}$ by

$$
P(\omega)= \begin{cases}-1+I_{\omega} & \text { if } \omega \text { is to the left of any element of } \\ & \kappa(\mathcal{L} \backslash\{\alpha\}), \\ I_{\kappa^{-1}}^{E_{1}}(\omega) & \text { if } \omega \in \kappa(\mathcal{L} \backslash\{\alpha\}), \\ {\left[I_{\alpha}^{E_{1}} \cap(s, t)\right] \cup(t, t+z)} & \text { if } \omega=\alpha^{\prime}, \\ t+z+I_{\omega} & \text { if } \omega \text { is to the right of } \alpha^{\prime} .\end{cases}
$$

A simple analysis shows that $P \in \mathcal{P}$. We set $E=\bigcup_{\omega \in \mathcal{K}} P(\omega)$. Then $E \in$ $\mathcal{E},(4.7)$ and (4.9) are satisfied, and it remains to verify the estimate (4.8). According to (3.9), we have

$$
\left\|f \chi_{(s, t)}\right\| \|_{E}=\sum_{k=0}^{\infty} \max _{\ell(\alpha)=k} \sup _{n \geq k} \frac{3^{n+1}}{2^{n}} \sum_{\substack{\ell(\omega)=n \\ \alpha \preceq \omega}} \int_{I_{\omega}^{E}}\left|f \chi_{(s, t)}\right| .
$$

The integrals vanish for all $\omega$ except $\omega=\alpha^{\prime}$ or $\omega \in \kappa(\mathcal{L})$, and

$$
\begin{aligned}
& \frac{3^{\ell(\omega)+1}}{2^{\ell(\omega)}} \int_{I_{\omega}^{E}}\left|f \chi_{(s, t)}\right|=\frac{3^{\ell(\omega)+1}}{2^{\ell(\omega)}} \int_{I_{\kappa_{1}-1}^{E_{1}}(\omega)}\left|f \chi_{(s, t)}\right| \quad \text { for } \omega \in \kappa(\mathcal{L}), \\
& \frac{3^{\ell\left(\alpha^{\prime}\right)+1}}{2^{\ell\left(\alpha^{\prime}\right)}} \int_{I_{\alpha^{\prime}}^{E}}\left|f \chi_{(s, t)}\right|=\left(\frac{3}{2}\right)^{j} \frac{3^{\ell(\alpha)+1}}{2^{\ell(\alpha)}} \int_{I_{\alpha}^{E_{1}}}\left|f \chi_{(s, t)}\right| .
\end{aligned}
$$


This immediately yields the inequality $\left\|f \chi_{(s, t)}\left|\left\|_{E} \geq\right\|\right| f \chi_{(s, t)} \mid\right\|_{E_{1}}$ and so, by (4.12), the estimate (4.8) holds.

Lemma 4.5. Let $E \in \mathcal{E}, \gamma \in \mathcal{K}, 0<s<t<1$ and $0<\tau<\theta / 2$. Let $f \in X$ be such that $\left\|f \chi_{(s, t)} \mid\right\|_{E} \geq \theta$. Then there exists a positive integer $N$ such that

$$
N \geq \ell(\gamma)
$$

and

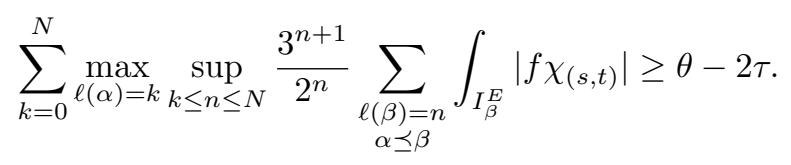

Proof. There exists a positive integer $N_{1} \geq \ell(\gamma)$ such that

$$
\sum_{k=0}^{N_{1}} \max _{\ell(\alpha)=k} \sup _{n \geq k} \frac{3^{n+1}}{2^{n}} \sum_{\substack{\ell(\beta)=n \\ \alpha \preceq \beta}} \int_{I_{\beta}^{E}}\left|f \chi_{(s, t)}\right| \geq \theta-\tau .
$$

For every $\alpha, \ell(\alpha)=k \leq N_{1}$, we can find an integer $N_{2}(k, \alpha) \geq N_{1}$ such that

$$
\sup _{k \leq n \leq N_{2}(k, \alpha)} \frac{3^{n+1}}{2^{n}} \sum_{\substack{\ell(\beta)=n \\ \alpha \preceq \beta}} \int_{I_{\beta}^{E}}\left|f \chi_{(s, t)}\right| \geq \sup _{n \geq k} \frac{3^{n+1}}{2^{n}} \sum_{\substack{\ell(\beta)=n \\ \alpha \preceq \beta}} \int_{I_{\beta}^{E}}\left|f \chi_{(s, t)}\right|-\frac{\tau}{2^{N_{1}+1}-1} .
$$

Setting $N=\max \left\{N_{2}(\alpha, k): 0 \leq k \leq N_{1}, \ell(\alpha)=k\right\}$ we obtain

$$
\begin{aligned}
\sum_{k=0}^{N} \max _{\ell(\alpha)=k} & \sup _{k \leq n \leq N} \frac{3^{n+1}}{2^{n}} \sum_{\substack{\ell(\beta)=n \\
\alpha \preceq \beta}} \int_{I_{\beta}^{E}}\left|f \chi_{(s, t)}\right| \\
& \geq \sum_{k=0}^{N_{1}} \max _{\ell(\alpha)=k} \sup _{\substack{k \leq n \leq N_{2}(\alpha, k) \\
2^{n}}} \frac{3^{n+1}}{\substack{\ell(\beta)=n \\
\alpha \preceq \beta}} \int_{I_{\beta}^{E}}\left|f \chi_{(s, t)}\right| \\
& \geq \sum_{k=0}^{N_{1}} \max _{\ell(\alpha)=k}\left(\sup _{n \geq k} \frac{3^{n+1}}{2^{n}} \sum_{\substack{\ell(\beta)=n \\
\alpha \preceq \beta}} \int_{I_{\beta}^{E}}\left|f \chi_{(s, t)}\right|-\frac{\tau}{2^{N_{1}+1}-1}\right) \\
& \geq \sum_{k=0}^{N_{1}} \max _{\ell(\alpha)=k} \sup _{n \geq k} \frac{3^{n+1}}{2^{n}} \sum_{\substack{\ell(\beta)=n \\
\alpha \preceq \beta}} \int_{I_{\beta}^{E}}\left|f \chi_{(s, t)}\right|-\tau \geq \theta-2 \tau .
\end{aligned}
$$


Lemma 4.6. Let $f \in X$ be such that

$$
\lim _{t \rightarrow 0+} \sup _{E \in \mathcal{E}}\left\|f \chi_{(0, t)} \mid\right\|_{E}=\eta>0 .
$$

Let $0<s<t<1, E \in \mathcal{E}$ and $\gamma \in \mathcal{K}$ satisfy (4.7) and

$$
\left\|f \chi_{(s, t)} \mid\right\|_{E}=\theta>0 .
$$

Then for every $\tau, 0<\tau<\min (\eta / 8, \theta / 2)$ there exist $F \in \mathcal{E}, s_{1} \in(0, s)$ and $\gamma_{1} \in \mathcal{K}$ such that $s_{1}=\inf I_{\gamma_{1}}^{F}$ and $\left\|f \chi_{\left(s_{1}, t\right)}\right\|_{F} \geq \theta+\frac{\eta}{2}-4 \tau$.

Proof. Let

$$
0<t_{1}<\min \left\{s / 7,3^{-N-2}\right\}
$$

where $N$ is the integer from Lemma 4.5. Since (4.6) holds with $t_{1}$ in place of $t$, from Lemma 4.4 we obtain $s_{1} \in\left(0, t_{1}\right), \gamma_{1} \in \mathcal{K}$ and $E_{1} \in \mathcal{E}$ such that

$$
\begin{gathered}
0<s_{1}:=\inf I_{\gamma_{1}}^{E_{1}}<t_{1}, \\
\left\|\mid f \chi_{\left(s_{1}, t_{1}\right)}\right\|_{E_{1}} \geq \frac{\eta}{2}-2 \tau \\
\sup I_{\omega}^{E_{1}} \leq 4 t_{1} \quad \text { and } \quad\left|I_{\omega}^{E_{1}}\right| \leq 3 t_{1} \quad \text { whenever } \quad I_{\omega}^{E_{1}} \cap\left(s_{1}, t_{1}\right) \neq \emptyset .
\end{gathered}
$$

Set

$$
\mathcal{L}=\left\{\omega: I_{\omega}^{E} \cap(s, t) \neq \emptyset\right\}, \quad \mathcal{L}_{1}=\left\{\omega: I_{\omega}^{E_{1}} \cap\left(s_{1}, t_{1}\right) \neq \emptyset\right\}
$$

and denote $\alpha=\left(a_{1}, \ldots, a_{k}\right)$ the (unique) minimal element of $\mathcal{L}_{1}$ with respect to the relation $\preceq$. By (4.23), (4.20) and (4.17), the inequalities

$$
3^{-\ell(\alpha)-1}=\left|I_{\alpha}^{E_{1}}\right| \leq 3 t_{1}<3^{-N-1} \leq 3^{-\ell(\gamma)-1}
$$

hold. Hence $\ell(\alpha) \geq \ell(\gamma)+1$. Let $\gamma=\left(c_{1}, \ldots, c_{j}\right)$. The first $k$ components of every element of $\mathcal{L}_{1}$ coincide with the components of $\alpha$ and we can define the injection $\kappa: \mathcal{L}_{1} \rightarrow \mathcal{K}$ by

$$
\begin{aligned}
\omega=\left(a_{1}, \ldots, a_{k}, b_{1}, \ldots, b_{n}\right) & \mapsto \kappa(\omega)=\left(c_{1}, \ldots, c_{j}, 0,1, \ldots, 1, b_{1}, \ldots, b_{n}\right), \\
\ell(\kappa(\omega)) & =\ell(\omega) .
\end{aligned}
$$

Every $\kappa(\omega)$ is to the left of every element of $\mathcal{L}$ because its $(j+1)$-th component is zero and, by (4.7), $\gamma$ is the left most element of $\mathcal{L}$. Denote by $\mathcal{H}_{1}$ the family of all multiindices which are to the left of every element of $\kappa\left(\mathcal{L}_{1}\right)$, by $\mathcal{H}_{2}$ the family of multiindices which are between $\kappa\left(\mathcal{L}_{1}\right)$ and $\mathcal{L}$, and by $\mathcal{H}_{3}$ the set of all multiindices which are to the right of every element of $\mathcal{L}$. 
Set $\delta=\left(c_{1}, \ldots, c_{j}, 0,1, \ldots, 1\right)$ with $\ell(\delta)=k+1, r=\inf \bigcup_{\delta \preceq \beta} I_{\beta}$ and define the mapping $P: \mathcal{K} \rightarrow \mathcal{F}$ by

$$
P(\omega)= \begin{cases}-1+I_{\omega} & \text { if } \omega \in \mathcal{H}_{1}, \\ I_{\kappa^{-1}(\omega)}^{E_{1}} & \text { if } \omega \in \kappa\left(\mathcal{L}_{1}\right), \\ -r+4 t_{1}+I_{\omega} & \text { if } \omega \in \mathcal{H}_{2}, \\ I_{\omega}^{E} & \text { if } \omega \in \mathcal{L}, \\ \sup E+I_{\omega} & \text { if } \omega \in \mathcal{H}_{3},\end{cases}
$$

where $I_{\omega}$ are the intervals from the construction of the classical Cantor set in Section 3. Set $F=\bigcup_{\omega \in \mathcal{K}} P(\omega)$. Then $P$ satisfies (3.1), $F$ is bounded and in order to show that $F \in \mathcal{E}$ we have to verify (3.2).

If $\beta$ is to the left of $\beta^{\prime}$ and both $\beta, \beta^{\prime}$ belong to one of the sets $\mathcal{H}_{1}, \kappa(\mathcal{L})$, $\mathcal{H}_{2}, \mathcal{L}$ or $\mathcal{H}_{3}$, then obviously $P(\beta)$ is to the left of $P\left(\beta^{\prime}\right)$. If $\beta \in \mathcal{H}_{1}$, then $P(\beta)=-1+I_{\omega} \subset(-1,0)$. If $\beta=\kappa(\omega)$ for some $\omega \in \mathcal{L}_{1}$, then $P(\beta)=I_{\omega}^{E_{1}} \subset$ $\left[s_{1}, 4 t_{1}\right]$ according to (4.21) and (4.23). If $\beta \in \mathcal{L}$, then $P(\beta) \subset[s$, sup $E]$. If $\beta \in \mathcal{H}_{3}$, then $P(\beta) \subset(\sup E, \sup E+1)$. Finally, let $\beta \in \mathcal{H}_{2}$. Since

$$
\mathcal{H}_{2} \subset\{\omega \in \mathcal{K}: \delta \preceq \omega\}
$$

we have $\inf P(\beta)>4 t_{1}$. On the other hand, by (4.24) and (4.23),

$$
\sup \bigcup_{\delta \preceq \omega} I_{\omega}-\inf \bigcup_{\delta \preceq \omega} I_{\omega}=\left|\bigcup_{\delta \preceq \omega} I_{\omega}\right|=3^{-\ell(\delta)}=3^{-k-1}=\left|I_{\alpha}^{E_{1}}\right| \leq 3 t_{1} .
$$

This yields $\sup P(\beta)=-r+4 t_{1}+\sup I_{\beta}<7 t_{1}<s$ by (4.20). All these relations together imply that $F$ satisfies (3.2).

Now, we can write

$$
\begin{aligned}
\left\|f \chi_{\left(s_{1}, t\right)} \mid\right\|_{F} \geq & \sum_{k=0}^{N} \max _{\ell(\alpha)=k} \sup _{k \leq n \leq N} \frac{3^{n+1}}{2^{n}} \sum_{\substack{\ell(\beta)=n \\
\alpha \preceq \beta}} \int_{I_{\beta}^{F}}\left|f \chi_{(s, t)}\right| \\
& +\sum_{k=N+1}^{\infty} \max _{\ell(\alpha)=k} \sup _{n \geq k} \frac{3^{n+1}}{2^{n}} \sum_{\substack{\ell(\beta)=n \\
\alpha \preceq \beta}} \int_{I_{\beta}^{F}}\left|f \chi_{\left(s_{1}, t_{1}\right)}\right|=A+B .
\end{aligned}
$$


Since $I_{\beta}^{F} \cap(s, t) \neq \emptyset$ if and only if $\beta \in \mathcal{L}$, we have

$$
\begin{aligned}
A & =\sum_{k=0}^{N} \max _{\ell(\alpha)=k} \sup _{k \leq n \leq N} \frac{3^{n+1}}{2^{n}} \sum_{\substack{\ell(\beta)=n \\
\alpha \preceq \beta}} \int_{I_{\beta}^{F}}\left|f \chi_{(s, t)}\right| \\
& =\sum_{k=0}^{N} \max _{\ell(\alpha)=k} \sup _{k \leq n \leq N} \frac{3^{n+1}}{2^{n}} \sum_{\substack{\ell(\beta)=n \\
\alpha \preceq \beta}} \int_{I_{\beta}^{E}}\left|f \chi_{(s, t)}\right|
\end{aligned}
$$

and so, according to the assumption (4.19) and Lemma 4.5, $A \geq \theta-2 \tau$. Using the definitions of $\mathcal{L}_{1}, \kappa$ and $P$ we obtain

$$
\begin{aligned}
B & =\sum_{k=N+1}^{\infty} \max _{\ell(\alpha)=k} \sup _{n \geq k} \frac{3^{n+1}}{2^{n}} \sum_{\substack{\ell(\beta)=n \\
\alpha \preceq \beta}} \int_{I_{\beta}^{F}}\left|f \chi_{\left(s_{1}, t_{1}\right)}\right| \\
& =\sum_{k=N+1}^{\infty} \max _{\ell(\alpha)=k} \sup _{n \geq k} \frac{3^{n+1}}{2^{n}} \sum_{\substack{\ell(\beta)=n \\
\alpha \preceq \beta}} \int_{I_{\beta}^{E_{1}}}\left|f \chi_{\left(s_{1}, t_{1}\right)}\right| .
\end{aligned}
$$

According to (4.23) and (4.20), for every $\beta \in \mathcal{L}_{1}$ we have $3^{-N-1}>3 t_{1} \geq$ $\left|I_{\beta}^{E_{1}}\right|=3^{-\ell(\beta)-1}$ and so, $\ell(\beta)>N$. Hence, by $(4.22)$,

$$
B=\sum_{k=0}^{\infty} \max _{\ell(\alpha)=k} \sup _{n \geq k} \frac{3^{n+1}}{2^{n}} \sum_{\substack{\ell(\beta)=n \\ \alpha \preceq \beta}} \int_{I_{\beta}^{E_{1}}}\left|f \chi_{\left(s_{1}, t_{1}\right)}\right|=\left\|f \chi_{\left(s_{1}, t_{1}\right)}\right\|_{E_{1}} \geq \frac{\eta}{2}-2 \tau .
$$

Thus, $\left\|f \chi_{\left(s_{1}, t\right)}\right\| \geq \geq A+B \geq \theta+\frac{\eta}{2}-4 \tau$.

Lemma 4.7. Every function $f \in X$ has continuous norm in $X$; i.e., $X_{c}=X$.

Proof. Our aim is to prove that for every $f \in X, \lim _{t \rightarrow 0+}\left\|f \chi_{(x-t, x+t) \cap(0,1)}\right\|=0$, $x \in[0,1]$ or, equivalently,

$$
\lim _{t \rightarrow 0+}\left\|f \chi_{(x, x+t)}\right\|=0, \quad x \in[0,1),
$$

and $\lim _{t \rightarrow 0+}\left\|f \chi_{(x-t, x)}\right\|=0, \quad x \in(0,1]$. We will prove only $(4.25)$; the other relation can be proved in the same way. Without loss of generality we can suppose that $x=0$ because $\left\|f \chi_{(x, x+t)}\right\|=\left\|g \chi_{(0, t)}\right\|$ for $g(\xi)=f(\xi+x)$, $0<\xi<1-x$. 
Assume, to the contrary, that $f \in X \backslash X_{c}, f \neq 0$; i.e., $f$ satisfies (4.18). Let $0<\tau<\eta / 22$. Then there exist $0<t<1$ and $E \in \mathcal{E}$ such that

$$
\eta \leq \sup _{E \in \mathcal{E}}\left\|f \chi_{(0, t)}\right\| \|_{E}<\eta+\tau .
$$

Let $s \in(0, t), \gamma \in \mathcal{K}$ and $E \in \mathcal{E}$ be from Lemma 4.4 and let $N$ be the corresponding integer from Lemma 4.5. Then the assumptions of Lemma 4.6 are satisfied with $\theta \geq \frac{\eta}{2}-2 \tau$ and so there exist $E_{1} \in \mathcal{E}, s_{1} \in(0, s)$ and $\gamma_{1} \in \mathcal{K}$ such that $s_{1}=\inf I_{\gamma_{1}}^{E_{1}}$ and $\left\|f \chi_{\left(s_{1}, t\right)}\right\|_{E_{1}} \geq \theta+\frac{\eta}{2}-4 \tau \geq \eta-6 \tau$. Hence the assumptions of Lemma 4.6 are satisfied with $s_{1}, E_{1}, \gamma_{1}$ and $\theta \geq \eta-6 \tau$, and there exist $E_{2} \in \mathcal{E}, s_{2} \in\left(0, s_{1}\right), \gamma_{2} \in \mathcal{K}$ such that $s_{2}=\inf I_{\gamma_{2}}^{E_{2}}$ and

$$
\left\|f \chi_{\left(s_{2}, t\right)} \mid\right\|_{E_{2}} \geq \theta+\frac{\eta}{2}-4 \tau \geq \frac{3}{2} \eta-10 \tau>\eta+\tau .
$$

This contradicts the assumption (4.26).

Remark. It is possible to repeat the last step in the proof ad libitum to get that $\|f\|=\infty$.

\section{References}

[1] C. Bennett and R. Sharpley, Interpolation of Operators, Pure Appl. Math., 129, Academic Press, 1988.

[2] D. E. Edmunds, J. Lang and A. Nekvinda, On $L^{p(x)}$-norms, Proc. R. Soc. London A, 455 (1999), 219-225.

[3] D. E. Edmunds, J. Rákosník, Density of smooth functions in $W^{k, p(x)}(\Omega)$, Proc. R. Soc. London, 437 (1992), 229-236.

[4] O. Kováčik, J. Rákosník, On spaces $L^{p(x)}$ and $W^{k, p(x)}$, Czechoslovak Math. J. 41 (1991), 592-618.

[5] Q. Lai and L. Pick, The Hardy operator, $L_{\infty}$ and BMO, J. London Math. Soc., 48 (1993), 167-177.

[6] J. Lang and A. Nekvinda, A difference between continuous and absolutely continuous norm in Banach function spaces, Czechoslovak Math. J., 47 (1997), 221-232.

[7] W. A. J. Luxemburg, Banach function spaces, Assen, Netherlands, 1955.

[8] A. C. Zaanen, Integration, North-Holland Publishing Company, Amsterdam, 1967. 